\title{
DISEASE IDENTIFICATION IN RABI CROP USING DEEP LEARNING
}

\author{
Simran Ramesh Amesar \\ Department of Information Technology \\ Thadomal Shahani Engineering College, University of Mumbai \\ Mumbai, Maharashtra, India
}

\begin{abstract}
The Indian economy is widely known to be majorly dependent on the farming efficiency. In order to satisfy the resident's interest and to give more advantages to ranchers, expanding crop creation is a critical undertaking currently. Harvest illnesses and nuisances assume a key job in diminishing yield creation and the quality of the crop. Huge amounts of potential crop outcome are lost along with major capital loss to the farmers due to this menace. This can lead to a grave situation of a famine consecutively leading to starvation or death. In this fashion, the identification of diseases is key to reduce the losses at an early stage. Manual discovery of maladies takes extra time and endeavors on the bigger territory of the homestead. Deep learning approach can be utilized to identify the infections and irritation, all the more precisely, on leaves and different parts of the harvest. The proposed strategy is useful in distinguishing crop illnesses similar to bugs. In this paper, the deep learning techniques related to diseases and pest detection has been reviewed and the deep learning model for programmed conclusion of yield ailments and bugs is proposed.
\end{abstract}

Keywords - Disease; crops; Inception; deep learning; convolutional neural networks (CNN)

\section{INTRODUCTION}

Farming is the pillar of the Indian economy. Colossal commercialization of farming makes a very negative impact on our condition. The utilization of compound pesticides has prompted huge degrees of synthetic development in our condition, in soil, water, air, in creatures and even in our own bodies. Counterfeit manures give on a momentary impact on profitability however a more drawn out term negative impact on the condition, where they stay for quite a long time in the wake of draining and running off, debasing ground water. Another negative impact of this pattern has been on the fortunes of the cultivating networks around the world. Despite this so-called increased productivity, farmers for all intents and purposes each nation around the world have seen a downturn in their fortunes. This is the place natural cultivating comes in. Natural cultivating has the ability to deal with every one of these issues. The focal action of natural cultivating depends on preparation, irritation and infection control. Plant malady identification through unaided eye perception of the indications on plant leaves, consolidate quickly expanding of multifaceted nature. Because of this intricacy and the enormous number of developed Crops and their current Phyto pathological issues, indeed, even experienced rural specialists and plant pathologists may frequently neglect to effectively analyze explicit infections, and are therefore prompted mixed up ends and concern arrangements. A robotized framework intended to help recognize plant maladies by the plant's appearance and visual side effects could be of incredible assistance to novices in the rural procedure. This will be demonstrated as a helpful method for farmers and will caution them at the perfect time before spreading the ailment over a huge zone.

The current technique, a large portion of the locales utilizing in India, is the manual discovery of the sickness by watching the fields persistently by the gathering of specialists. It is an extremely meticulous undertaking and less exact when observing the bigger zone of the ranch. A few farmers are not notable to all the maladies, don't have appropriate offices and ordinarily likewise not mindful of how to contact a specialist to get a proposal. At times even experienced agronomists regularly neglect to effectively analyze the harvest sickness. As the side effects of ailments are by and large showing up on the diverse piece of the harvests and can be distinguished outwardly, the pictures of those tainted parts can be utilized for location. Along these lines, the programmed discovery of the sickness from the pictures is significant to help the master as it is progressively precise and takes less time and endeavors. In enormous scope cultivating, the proposed plan could be joined with the IoT gadgets to accurately distinguish the illness, utilizing persistent picture catching. All the gathered pictures at that point can be handled utilizing the profound learning approach. Convolutional Neural Network is exceptionally proficient as far as handling a lot of information in less time. The calibrating of the pre-prepared CNN model is utilized for handling the picture information of leaf, stem, root, organic products and so on parts of the yield which are tainted from ailments and nuisance. 


\section{RELATED WORKS}

In paper [1], the author discussed and to play out a review of 40 research endeavors that utilize profound learning methods, applied to different rural and food production challenges. Inspect the specific agrarian issues under examination, the explicit models and structures utilized the sources, nature also, pre-processing of data utilized, and the general execution accomplished by the metrics utilized at each work under study. In addition, study correlations of deep learning with other existing well-known methods, in regard to contrasts in classification or regression performance. Discoveries show that deep learning gives high accuracy, beating existing normally utilized strategies.

In paper [2], the author compared two deep learning models AlexNet and GoogLeNet for Image-Based plant infection detection. They chose a dataset of 54,306 pictures of solid and sick leaves of 14 harvests and 26 illnesses. They have performed experiments with a dataset by taking shading, gray scale and divided leaf pictures. They have likewise taken diverse preparing, training-testing ratios of images. GoogLeNet with Transfer learning and color crop images, which are in the extent of $80 \%-20 \%$ training-testing proportion has accomplished a precision of $99.34 \%$. The outcome shows that GoogLeNet performs better than AlexNet in their exploration work.

In paper [3], the author utilized deep learning to figure out how to evaluate the infection's seriousness dependent on the images. Disease seriousness estimation utilizing conventional picture preparing methods requires additional time and ability on account of hand-created includes should be provided to the model. They have used the apple black rot images of the Plant Village dataset, which are additionally clarified with the four phases to distinguish the stage of a sickness. The stages are sound, early, middle and end stage. They have looked at the shallower network which is prepared from the scratch and deeper network utilizing transfer learning. The proposed work has an image preprocessing stage which incorporates picture resizing for various models and growths like turn, shearing, zooming and so forth. They have taken VGGNet, Inception-v3 and ResNet50 models for move learning. The VGG16 model with move learning plays out the best and gets the exactness of $90.4 \%$. They proposed that the model can be related with the treatment suggestion and yield expectation.

In paper [4], a neural network is used to distinguish the ailment. On the off chance that leaf is tainted, further preparing is done to distinguish the sickness. Hereditary calculation is utilized alongside SVM to optimize misfortune and distinguish the kind of ailment. This paper states a strategy for enhancement of loss function utilizing genetic algorithms, which is like the hypothesis of natural selection where just solid boundaries endure.

In this paper [5], Rangarajan et al. have performed early analysis of tomato disease utilizing profound learning calculation for pre-prepared systems. They have taken 6 maladies of the tomato and 13,262 fragmented images from the PlantVillage database. AlexNet and VGG16 models were utilized in the analysis of the malady. The exchange learning approach is utilized for the preparing of the two models. The AlexNet model accomplishes exactness of $97.49 \%$ and VGG16 accomplishes $97.23 \%$. They have explored different avenues regarding the hyper boundaries like minibatch size, bias learning rate and weight to assess the execution.

\section{MeTHODOLOGY}

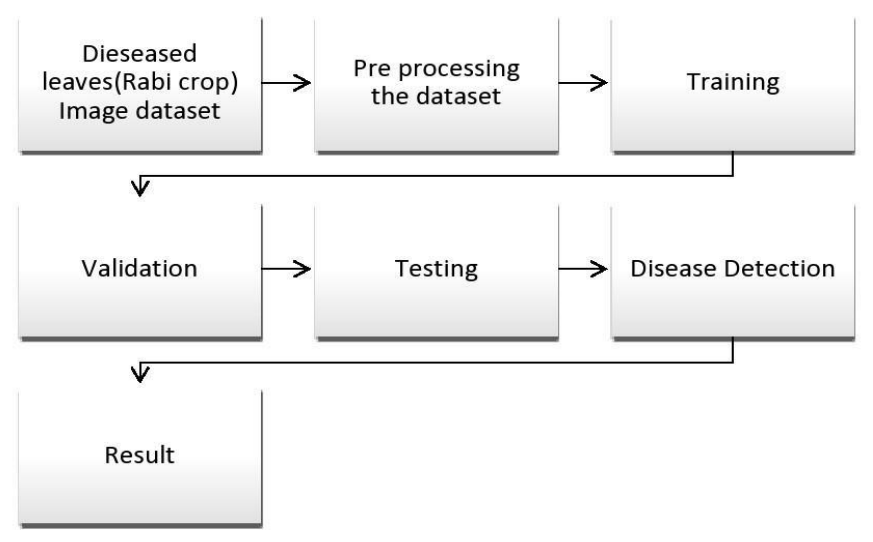

Fig.1. Flow of the strategy

\section{A. Dataset description}

Dataset is one among the foremost important and vital aspects of any research study. The info for our study is obtained from Kaggle, a web community of knowledge scientists and machine learning practitioners. We chose Plantvillage dataset, which may be a publicly open source dataset from Kaggle solely made for the aim of Disease detection system. The dataset consists of maladies plant leaf images and corresponding labels. This dataset contains 20,639 images of crop leaves which is assessed into 15 different classes. Note that, these data accompany predefined training and test subsets. After the dataset was loaded and every one the required packages imported, we proceeded on splitting the info into testing set and training set i.e. $80 \%$ of the info is for the training set and therefore the remaining 20\% for the test (more precisely, 16511 training images and 4128 images for the test) so as to facilitate efficient and quick handling of knowledge. 
< Pepper_bell__Bacterial_spot (997 files)
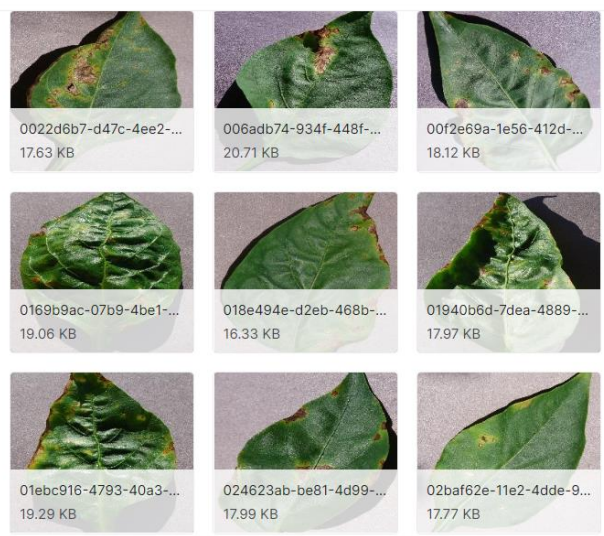

$16.33 \mathrm{~KB}$

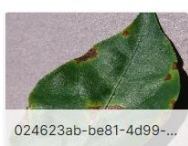

$17.99 \mathrm{~KB}$

. Fig.2. Examples of Plant leaf images

\section{B. Pre-processing and Augmentation}

After the selection of the dataset, the procedure of preprocessing takes place which comprises routines like cropping of image, flipping horizontally/vertically, altering saturation or other parameters like contrast etc. We have cropped the images to a uniform size in our project. In addition to this we have also used Data augmentation, which is a modus operandi to artificially create new training data from existing training data as the effectiveness of deep learning neural networks often ameliorate with the amount of data available. We have used image augmentation, which is a type of data augmentation that includes creating transformed versions of images in the training dataset that belong to the same class as the image in the original.

\section{Models}

\section{Convolutional Neural Network}

A convolutional neural network or CNN is a subset (class) of deep learning which is used in the field of image classification. Basically, a convolutional layer is used to test and train the data. The input image will go through a sequence of convolution layers which have inbuilt filters (Kernels), Pooling, FC (fully connected layers) in them. After the image is gone through it a function called SoftMax is integrated which helps in cataloguing the object into a particular class by providing probabilistic values.

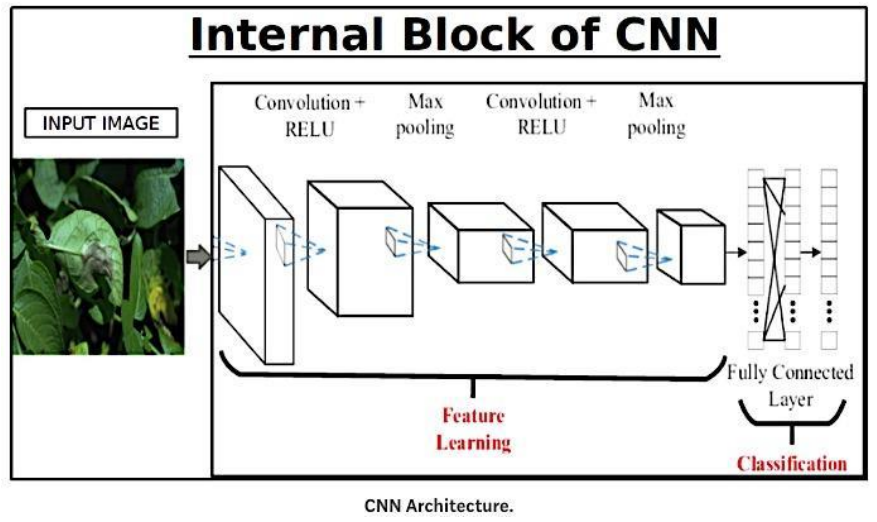

Fig.3. Internal Block of CNN

Convolutional layer- It is the first layer of the network. It comprises of filters called as kernels which when applied on the data (via matrix multiplication) produces a feature map, which describes the features of the image

Activation layer- This layer contains non linearity via functions like Relu, sigmoid etc. As the name suggests it essentially means whether a function will be initiated or not

Stride and Padding- Stride states by what value (default 1 ) will the convolution filter move at each step. Bigger strides mean less overlaps which gives rise to smaller feature maps as we are discarding budding locations. Dimensionality is maintained through padding it.

Maximum pooling- This function is basically used to lower the dimensionality and parameters by choosing the greatest element out of the feature map. It is used to tackle over fitting. In supplement to these we have used functionalities like dropout and batch normalization to increase the accuracy.

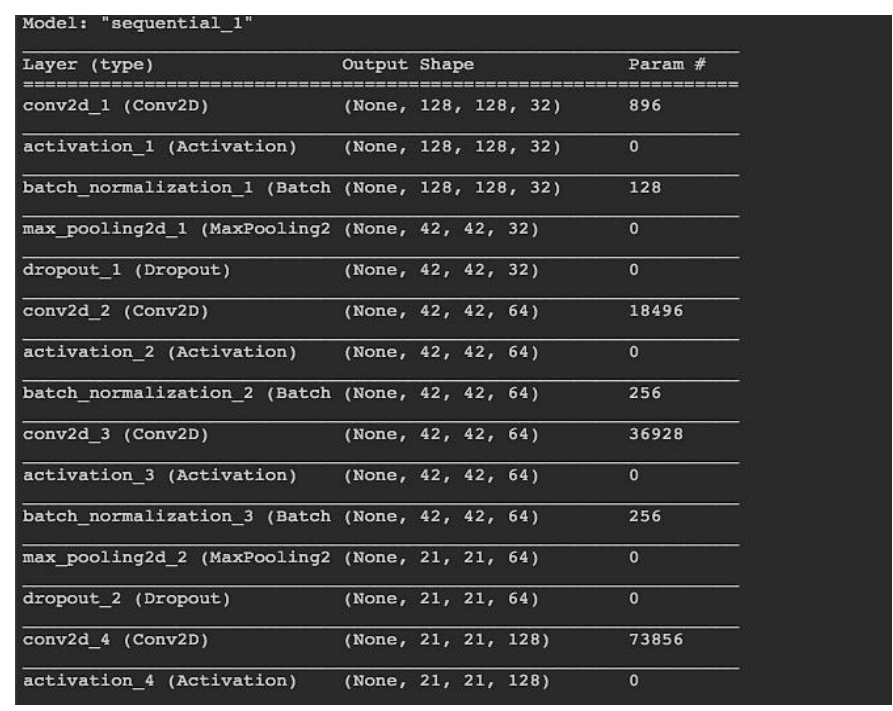

Fig.4. Model Summary 


\section{International Journal of Engineering Applied Sciences and Technology, 2020 \\ Vol. 5, Issue 6, ISSN No. 2455-2143, Pages 249-256 \\ Published Online October 2020 in IJEAST (http://www.ijeast.com)}

In the project, in addition to the convolutional network model we have used the following models to predict the plant disease:

\section{Inception}

Inception $\mathrm{v} 3$ is an extensively operated $\mathrm{CNN}$ based image detection model. It has been illustrated to attain an accuracy greater than $78.1 \%$ on the ImageNet dataset. The model is essentially based on the apotheosis of a plethora of designs and ideas of numerous researchers. The idea was first cultivated in the original paper: "Rethinking the Inception Architecture for Computer Vision" by Szegedy, et. al.

It is a 48-layer deep model which consists of both symmetric and asymmetric structures, and has functions like convolutions, pooling, dropouts, and fully connected layers. Batch normalization is used throughout the model copiously. It can essentially be pre-trained on the ImageNet, (which contains millions of images) and provides high accuracy.

The following figure shows an HLL diagram of an inception model.

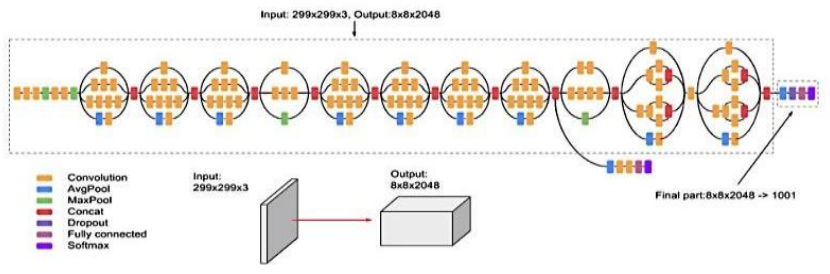

Fig.5. HLL diagram of Inception model

\section{ResNet}

Residual Neural Network by Kaiming He et al introduced an architecture with "skip connections" which features heavy batch normalization.

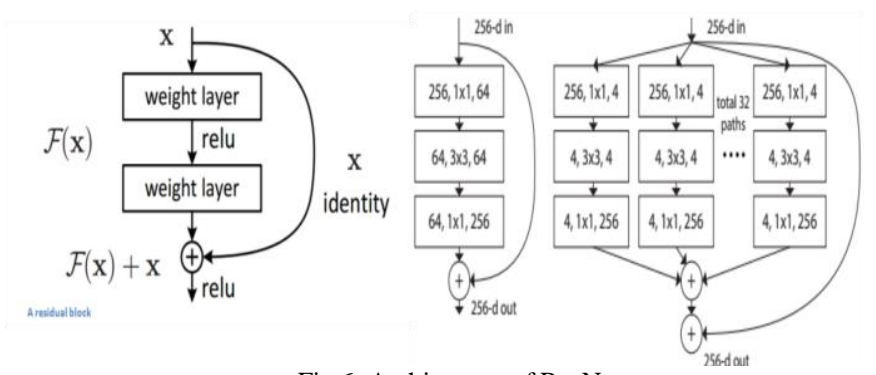

Fig.6. Architecture of ResNet

\section{AlexNet}

The AlexNet follows the same design pattern as the LeNet-5 architecture. The LeNet-5 variants are usually a set of convolution layers along with one or more fully connected layers. The convolution layers may or may not have a normalization layer and a pooling layer following it, and all the layers in the network usually have ReLu non-linear activation units associated with them. AlexNet comprises 5 convolution layers, along with 3 fully connected layers, and ending with a SoftMax layer.

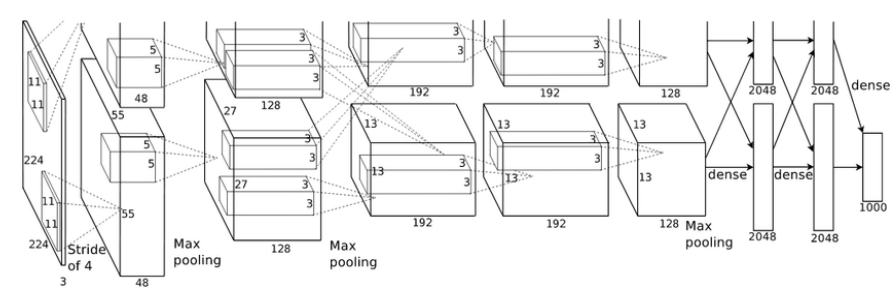

Fig.7. Architecture of AlexNet

\section{DenseNet}

In DenseNet, each of the layers gets additional inputs from all preceding layers and passes on its own feature-maps to all or any subsequent layers. Concatenation is employed within the layers. Each and every layer is receiving a "collective knowledge" from all preceding layers. Since each layer receives feature maps from all preceding layers, networks are often thinner and compact, i.e. number of channels are often fewer. the expansion rate $\mathrm{k}$ is that the additional number of channels for every layer. So, DenseNet has computational efficiency and memory efficiency.

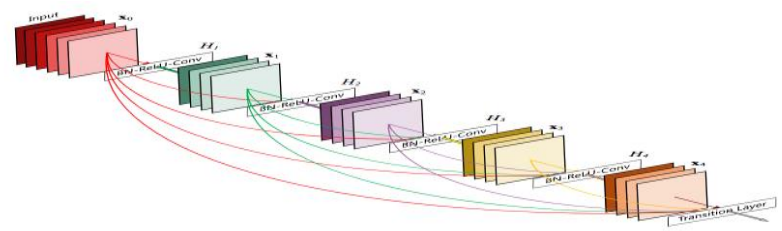

Fig.8. Architecture of DenseNet

Vgg16

The input to convolution 1 layer is of fixed size $224 \times 224$ image. This is passed through a pile of convolutional layers, where the filters were used with a very small receptive field. In the configurations, it also utilizes $1 \times 1$ convolution filter, which can be seen as a linear transformation of the input channels (following non-linearity).

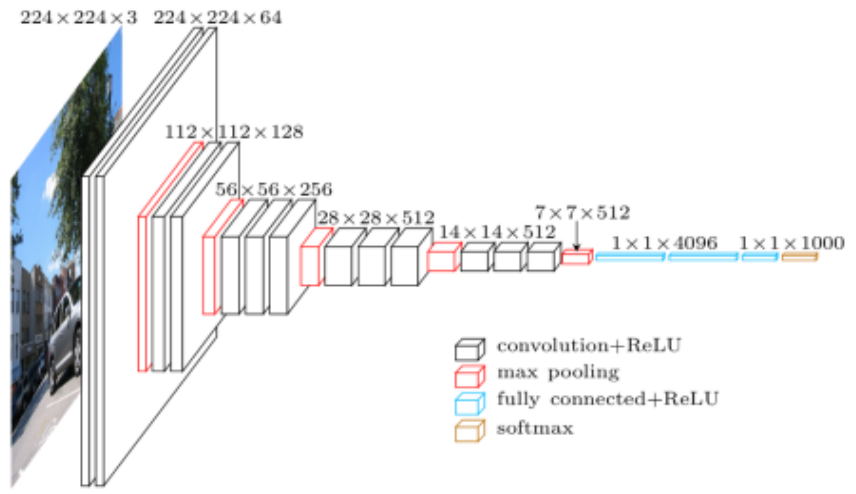

Fig.9. Architecture of Vgg16 


\section{Evaluation Criteria}

The Classification model's value and efficiency were measured utilizing the conventional measurements of precision, accuracy, recall, and F1 score. Accuracy is the calculation of the model's redress predictions and all overall expectations.

\section{EXPERIMENTAL RESULTS}

\section{Inception}

In Inception, after combining the complete dataset, it was split in a 70:30 ratio, with $70 \%$ used for Training while $30 \%$ was used for testing. The original image was resized to $150 \mathrm{x} 150 \mathrm{x} 3$ and then given as input, which yielded the following results after 5 epochs. The Batch size was 32 and Adam optimizer was used.
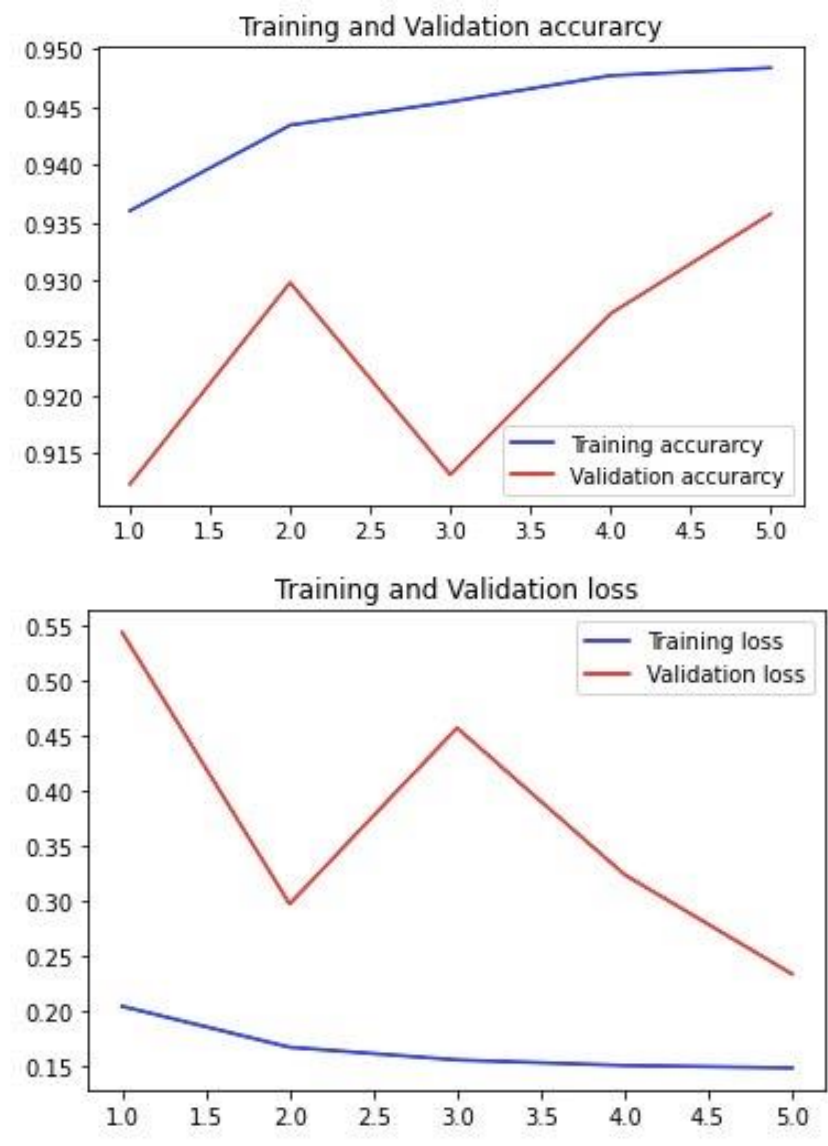

Fig.10. Training and Validation loss and accuracy

\begin{tabular}{|l|c|}
\hline Precision Score & 0.855422 \\
\hline Recall Score & 0.845238 \\
\hline F1 Score & 0.850299 \\
\hline
\end{tabular}

Table 1. Results- Inception model

\section{ResNet}

In Resnet, the dataset was divided into 70:30 ratio, the model was run for 5 Epochs with Batch size 32 and Adam optimizer.
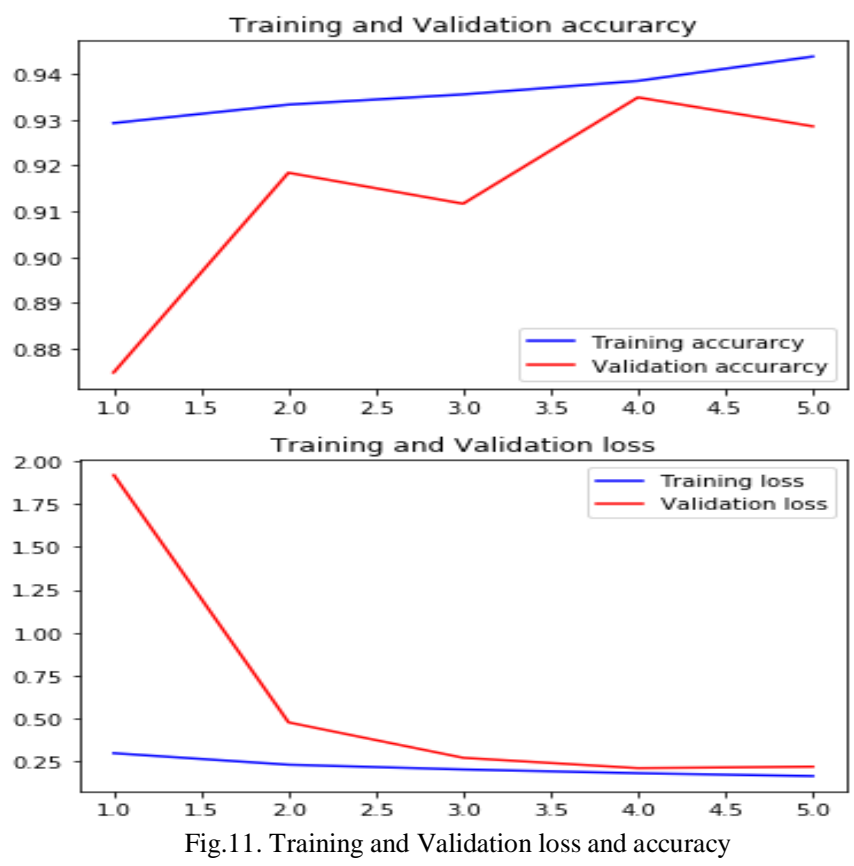

\begin{tabular}{|l|c|}
\hline Precision Score & 0.850412 \\
\hline Recall Score & 0.854132 \\
\hline F1 Score & 0.852422 \\
\hline
\end{tabular}

Table 2. Results- ResNet model

\section{AlexNet}

AlexNet had a batch size of 32, for 15 epochs and used Adam optimizer. The following shows the Training and Validation loss and accuracy, with the precision score, recall score and F1 score. 
International Journal of Engineering Applied Sciences and Technology, 2020

Vol. 5, Issue 6, ISSN No. 2455-2143, Pages 249-256

Published Online October 2020 in IJEAST (http://www.ijeast.com)
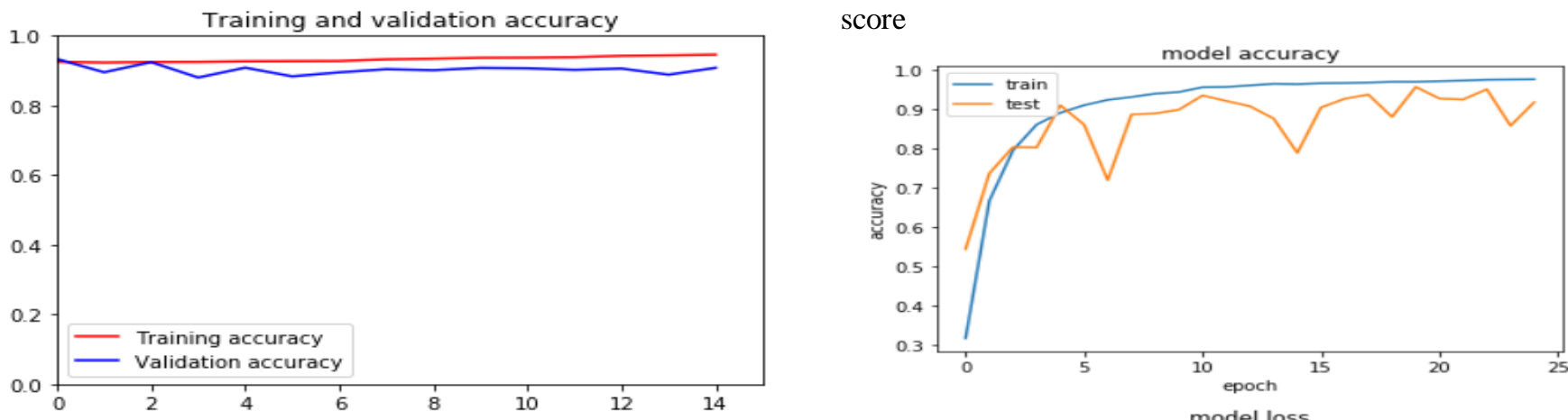

Training and validation loss
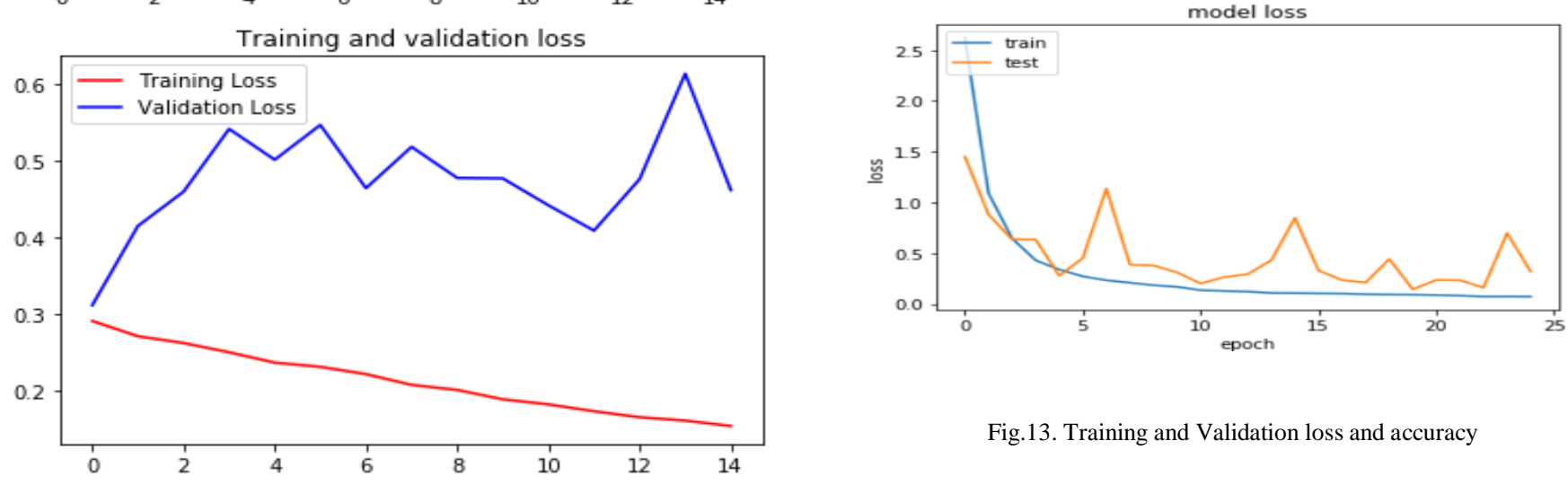

Fig.13. Training and Validation loss and accuracy

Fig.12. Training and Validation loss and accuracy

\begin{tabular}{|l|c|}
\hline Precision Score & 0.856822 \\
\hline Recall Score & 0.852920 \\
\hline F1 Score & 0.850334 \\
\hline
\end{tabular}

\begin{tabular}{|l|c|}
\hline Precision Score & 0.850394 \\
\hline Recall Score & 0.857143 \\
\hline F1 Score & 0.850334 \\
\hline
\end{tabular}

Table 4. Results- DenseNet

Table 3. Results- AlexNet

\section{VGG16}

In VGG16, the model had the batch size of 32, for 30 epochs and used Adam Optimizer. The following shows the Training and Validation loss and accuracy, with the precision score, recall score and F1 score. 
International Journal of Engineering Applied Sciences and Technology, 2020

Vol. 5, Issue 6, ISSN No. 2455-2143, Pages 249-256

Published Online October 2020 in IJEAST (http://www.ijeast.com)
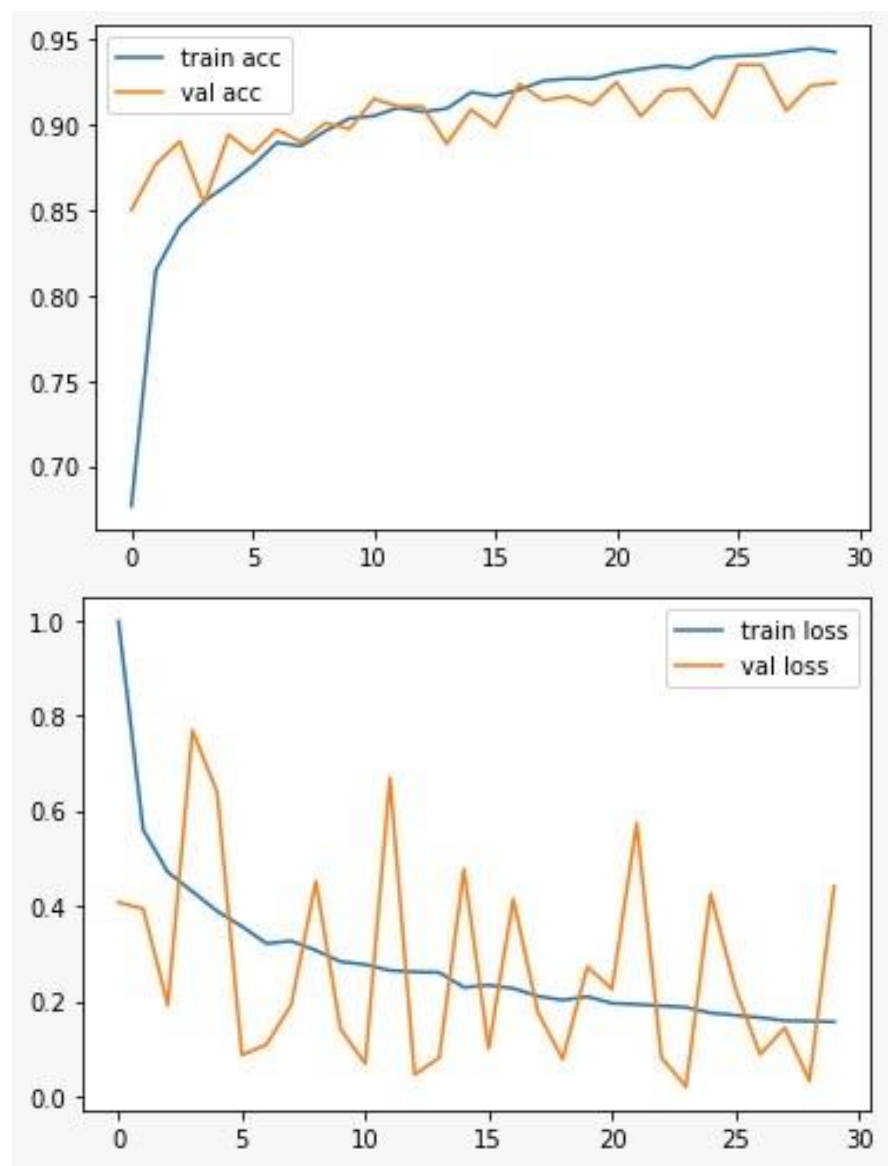

Fig.14. Training and Validation loss and accuracy

\begin{tabular}{|l|l|}
\hline Precision Score & 0.071384 \\
\hline Recall Score & 0.071310 \\
\hline F1 Score & 0.0710975 \\
\hline
\end{tabular}

Table 5. Results- Vgg16 model

Convolutional Neural Networks

In CNN, we divided the data into a 70:30 ratio after combining the entire dataset. $70 \%$ was used for training and $30 \%$ for testing. Epochs was 25 with Optimizer Adam. Batch size of 32 was taken to improve the models learning ability.
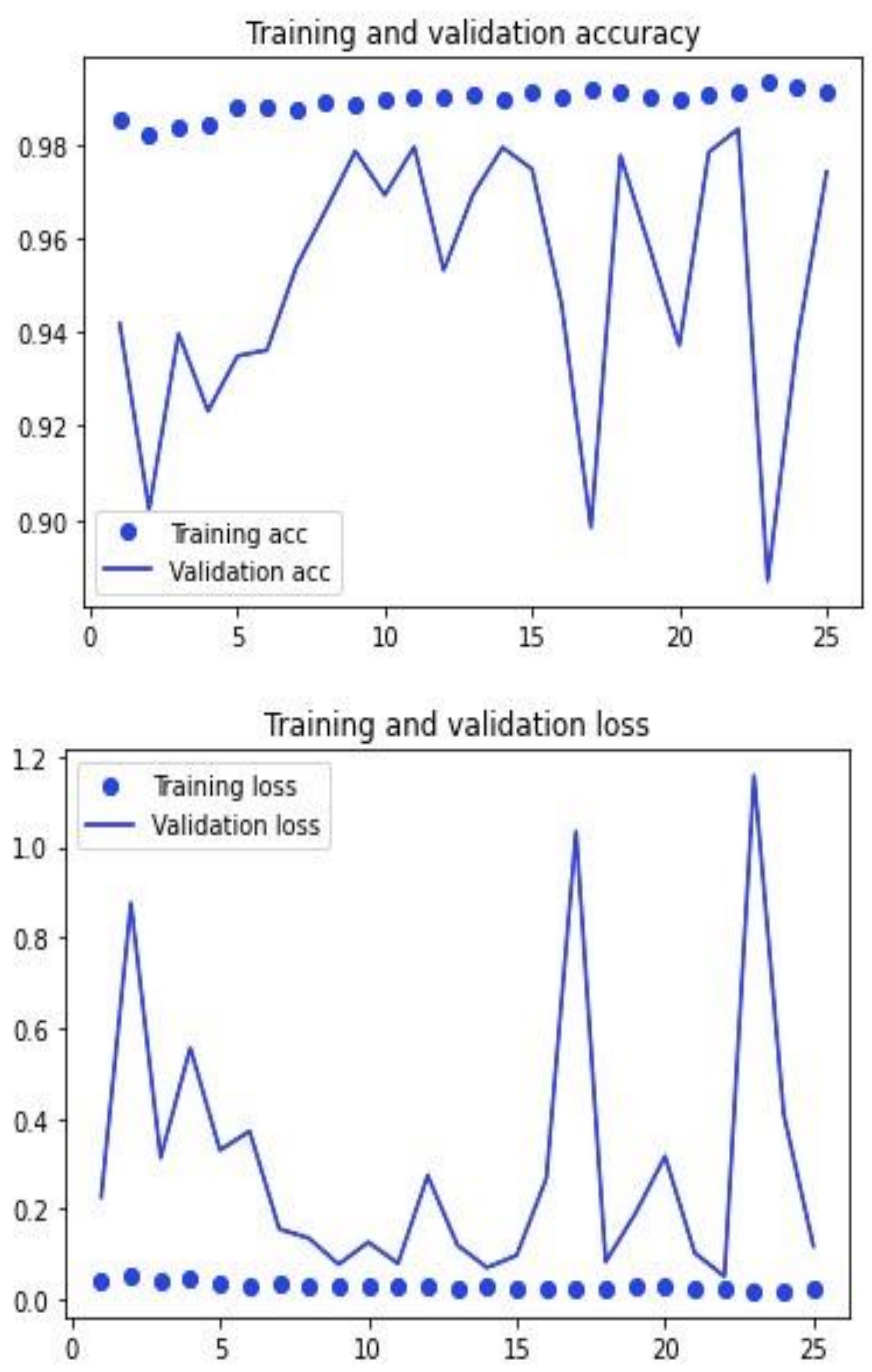

Fig.15. Training and Validation loss and accuracy

\begin{tabular}{|l|l|}
\hline Precision Score & 0.875682 \\
\hline Recall Score & 0.863786 \\
\hline F1 Score & 0.861295 \\
\hline
\end{tabular}

Table 6. Results- CNN model

V. RESULTS

The results of the above discussed models can be summarized in Table below. The Convolutional neural network model gives us the best $\mathrm{f} 1$ score of $86.1 \%$, Recall Score of $86.37 \%$, Test Accuracy of $97.41 \%$ and Precision of $87.57 \%$. 


\begin{tabular}{|l|l|l|l|l|}
\hline Model & Precision Score & Recall Score & F1 Score & Accuracy \\
\hline CNN & 0.875682 & 0.863786 & 0.861295 & 97.41 \\
\hline Vgg16 & 0.071384 & 0.071310 & 0.0710975 & 92.42 \\
\hline ResNet & 0.850412 & 0.854132 & 0.852422 & 93.51 \\
\hline DenseNet & 0.850394 & 0.857143 & 0.850334 & 91.80 \\
\hline AlexNet & 0.856822 & 0.852920 & 0.850334 & 90.79 \\
\hline Inception & 0.855422 & 0.845238 & 0.850299 & 93.08 \\
\hline
\end{tabular}

Table 7. Results

\section{CONCLUSION}

Diseases in plants are a major threat to food supply, as they cause reduction in quantity as well as quality of the agricultural produce. Our solution demonstrates the technical feasibility of deep learning using convolutional neural networks trained through image classification to classify crop species and disease status of different classes, achieving a test accuracy of 97.41\%. With minimal computational efforts, the most optimum results were obtained, which also shows the efficiency in recognition and classification successfully between healthy leaves and different diseases, which were detected at the initial stage. A plant disease recognition system can work as a universal detector recognizing general abnormalities on the leaves provides clear benefit in monitoring of large fields, as this approach provides a chance to discover diseases at an early stage, reduces the requirement of experts and prevents huge losses.

\section{REFERENCE}

[1] Andreas Kamilaris, Francesc X. Prenafeta-Boldu Deep learning in agriculture: (2018) A survey, Computers and Electronics in Agriculture 147 70-90, 10.1016/j.compag.2018.02.016

[2] Mohanty, Sharada P., David P. Hughes, and Marcel Salathé,( 2016) "Using deep learning for image-based plant disease detection." Frontiers in plant science 7, p. $1419,10.3389 /$ fpls.2016.01419

[3] Wang, Guan, Yu Sun, and Jianxin Wang,(2017) "Automatic image-based plant disease severity estimation using deep learning," Computational intelligence and neuroscience. 10.1155/2017/2917536
[4] Naik, M.R., Sivappagari, C., (2016) "Plant Leaf and Disease Detection by Using HSV Features and SVM," IJESC, Volume 6 Issue No.12.

[5] Rangarajan, Aravind Krishnaswamy, Raja Purushothaman, and Aniirudh Ramesh.( 2018) "Tomato crop disease classification using pre-trained deep learning algorithm." Procedia computer science 133. pp. 10401047, 10.1016/j.procs.2018.07.070

[6] LeCun Y., Bottou L., Orr G.B., Müller K.R. (1998) Efficient BackProp. In: Orr G.B., Müller KR. (eds) Neural Networks: Tricks of the Trade. Lecture Notes in Computer Science, vol 1524. Springer, Berlin, Heidelberg, https://doi.org/10.1007/3-540-49430-8 2

[7] K. He, X. Zhang, S. Ren and J. Sun, "Deep Residual Learning for Image Recognition," 2016 IEEE Conference on Computer Vision and Pattern Recognition (CVPR), Las Vegas, NV, 2016, pp. 770-778, doi: 10.1109/CVPR.2016.90.

[8] LeCun, Y., Bengio, Y. \& Hinton, (2015) G. Deep learning. Nature 521, 436-444, https://doi.org/10.1038/nature14539

[9] Srdjan Sladojevic, Marko Arsenovic, Andras Anderla, Dubravko Culibrk, Darko Stefanovic, "Deep Neural Networks Based Recognition of Plant Diseases by Leaf Image Classification", Computational Intelligence and Neuroscience, vol. 2016, Article

ID 3289801, 11 pages, 2016. https://doi.org/10.1155/2016 /3289801

[10] Kaur, S., Pandey, S., \& Goel, S. (2018). Plants Disease Identification and Classification Through Leaf Images: A Survey. Archives of Computational Methods in Engineering. doi:10.1007/s11831-018-9255-6

[11] Saleem, Potgieter, \& Mahmood Arif. (2019). Plant Disease Detection and Classification by Deep Learning. Plants, 8(11), 468. doi:10.3390/plants8110468

[12] C. Szegedy, W. Liu, Y. Jia, P. Sarmanet, S. Reed, D. Anguelov, D. Erhan, V. Canhoucke and A. Rabinovich, "Going Deeper with Convolutions," eprint arXiv:1409.4842, pp. 1-12, 2014

[13] Durmus, H., Gunes, E. O., \& Kirci, M. (2017). Disease detection on the leaves of the tomato plants by using deep learning. 2017 6th International Conference on AgroGeoinformatics. doi:10.1109/agrogeoinformatics.2017.8047016 